\title{
New Higgs Production Mechanism in Composite Higgs Models
}

\author{
Adrián Carmona, Mikael Chala and José Santiago \\ CAFPE and Dpto. de Física Teórica y del Cosmos \\ Universidad de Granada, E-18071, Granada, Spain
}

\begin{abstract}
Composite Higgs models are only now starting to be probed at the Large Hadron Collider by Higgs searches. We point out that new resonances, abundant in these models, can mediate new production mechanisms for the composite Higgs. The new channels involve the exchange of a massive color octet and single production of new fermion resonances with subsequent decays into the Higgs and a Standard Model quark. The sizable cross section and very distinctive kinematics allow for a very clean extraction of the signal over the background with high statistical significance. Heavy gluon masses up to $2.8 \mathrm{TeV}$ can be probed with data collected during 2012 and up to $5 \mathrm{TeV}$ after the energy upgrade to $\sqrt{s}=14 \mathrm{TeV}$.
\end{abstract}




\section{Contents}

1 Introduction 1

2 The Model 3

3 Experimental Constraints $\quad 7$

$\begin{array}{lll}3.1 & \text { Higgs searches } & 7\end{array}$

$\begin{array}{lll}3.2 & \text { Vector-like quark searches } & 8\end{array}$

$3.3 t \bar{t}$ resonance searches 8

3.4 Di-jet searches 9

4 Searches for New Composite Higgs Production Mechanisms 10

$\begin{array}{lll}4.1 & H t \bar{t} \text { analysis: low energy phase } & 12\end{array}$

$\begin{array}{lll}4.2 H t \bar{t} \text { analysis: high energy phase } & 14\end{array}$

$\begin{array}{lll}4.3 H j \text { analysis: high energy phase } & 15\end{array}$

5 Results and Discussion $\quad 16$

$\begin{array}{lll}5.1 H t \bar{t} \text { channel: MFV scenario } & 17\end{array}$

$\begin{array}{ll}5.2 H j j \text { channel: MFV scenario } & 19\end{array}$

$\begin{array}{lll}5.3 H t \bar{t} \text { channel: anarchy scenario } & 20\end{array}$

6 Conclusions 21

\section{Introduction}

Models of strong electroweak symmetry breaking (EWSB), and in particular composite Higgs models [1-3] in which the Higgs is a pseudo-Goldstone boson of a global symmetry of the composite sector, are very attractive candidates for a natural realization of EWSB. Despite the expected abundance of new resonances at the $\mathrm{TeV}$ scale in these models, because of the strong coupling in the composite sector, electroweak precision tests require these new resonances to be typically beyond the reach of current searches at the Large Hadron Collider (LHC) [4-6]. The Higgs couplings are modified in these models and thanks to the current "Higgs effort" the LHC community is going through, the first non-trivial LHC constraints on minimal composite Higgs models come from Higgs searches, even for a moderate degree of compositeness [7-11] (see also [12]). ${ }^{1}$

New composite states, even if they are heavy enough to escape standard analyses, could be eventually accessible with more ingenuous searches. For instance, it has been

\footnotetext{
${ }^{1}$ Genuine tests of Higgs compositeness, based on longitudinal gauge boson and Higgs scattering will require a much longer wait [13].
} 
recently emphasized that new color octet resonances in composite models could be more efficiently searched for through their decay into a massive fermion resonance and a Standard Model (SM) quark [14-17] (this was first pointed out, but not fully explored in this context, in [18]). These studies considered the channels in which the new fermion resonance decays into an electroweak gauge boson and a SM quark. However, a sizable fraction of the heavy fermion resonances will decay into a SM quark and the composite Higgs, thus making this process a new production mechanism for the composite Higgs. We explore such production mechanism in this article (see contribution 12 of [19] for preliminary results in the $H t \bar{t}$ channel). The resulting final state is of the form $H q \bar{q}$, with $q$ any SM quark. The corresponding production cross-sections are sizable but not dramatic. However, the very distinctive kinematics make the signal cross section stand out of the background even with very simple (and therefore quite robust) analyses. In the following we will call the new fermion resonances vector-like quarks and the new color octet boson resonances heavy gluons. This just reflects their nature and emphasizes that the new production mechanism occurs in more general models than composite Higgs models. New color octet resonances play no role in models of strong EWSB and are usually disregarded. However, in the context of partial compositeness $[20,21]$, that we adopt in this article, their presence is almost unavoidable. ${ }^{2}$ Indeed, partial compositeness implies a linear coupling of elementary fields to composite operators of the strongly coupled sector. In particular, the linear coupling of quarks implies that the strong sector must have a global $S U(3)_{c}$ symmetry. The two point correlators of the corresponding conserved currents will include vector resonances in the octet representation with which the SM gluons will mix. Thus, although we are motivated by holographic models of strong EWSB, the presence of new colored resonances is more general than that in the context of models with partial compositeness.

Higgs production through the decay of new vector-like quarks has been considered in the literature for quite some time [24-29]. In most cases the process considered is pair production of the new vector-like quarks followed by decays into electroweak and/or Higgs bosons and SM quarks. This mechanism is essentially model independent as the production is dominated by QCD and the equivalence theorem guarantees that $\sim 1 / 4$ of the produced quarks decay into the Higgs and a SM quark. The number of vector-like quarks being essentially the only free parameter. ${ }^{3}$ An alternative channel has been considered in [30, 31]. It consists of electroweak single production of new vector-like quarks with subsequent decay into the Higgs and a SM quark. This channel is more model dependent as the production cross section depends on unknown electroweak couplings of the heavy quark. All these processes are also present in the models we are considering. The interesting fact is that all three are sensitive to different couplings and it is therefore important to study all of them independently as they can provide very useful information on the properties of the composite sector.

The outline of the article is as follows. We introduce the model and describe the main

\footnotetext{
${ }^{2}$ Similar arguments have been used in [22, 23].

${ }^{3}$ Depending on the new quark quantum numbers, branching fractions into Higgs could be up to 1 but only at the expense of having other quarks for which such channel is forbidden in such a way that the global $1 / 4$ factor is approximately preserved.
} 
features relevant for the new Higgs production mechanism in section 2. Current constraints on the model are described in section 3. The analyses we propose to search for these new channels are described in section 4 and we discuss the results in section 5 . We summarize our results in section 6 .

\section{The Model}

The new Higgs production mechanism we want to study consists of the single production of a new vector-like quark (in association with a SM quark) mediated by the exchange of a heavy gluon and with subsequent decay in a SM quark and the Higgs. Thus, the only relevant ingredients are new vector-like quarks and new massive gluons. This mechanism is therefore common to many models of strong EWSB independently of whether the Higgs is a pseudo-Goldstone boson or not. However, in order to be able to give quantitative results, we focus in this article on the minimal composite Higgs model based on the $S O(5) / S O(4)$ coset with composite fermions transforming in the fundamental (5) representation of $S O(5)$, denoted by $\mathrm{MCHM}_{5}[32,33]$. The coset structure and the fermion quantum numbers fix the Higgs couplings to the SM particles (assuming the composite states to be heavy enough so that mixing effects can be neglected) in terms of a single parameter

$$
\xi=\frac{v^{2}}{f^{2}},
$$

where $v=2 m_{W} / g \approx 246 \mathrm{GeV}$ (with $m_{W}$ the $W$ mass and $g$ the $S U(2)_{L}$ coupling constant) and $f$ is the decay constant of the composite sector. In the $\mathrm{MCHM}_{5}$ model, the ratios of the tree level couplings of the Higgs to two SM particles to the corresponding SM coupling read (see for instance $[7,13]$ ):

$$
R_{H V V} \equiv \frac{g_{H V V}}{g_{H V V}^{\mathrm{SM}}}=\sqrt{1-\xi}, \quad R_{H f f} \equiv \frac{g_{H f f}}{g_{H f f}^{\mathrm{SM}}}=\frac{1-2 \xi}{\sqrt{1-\xi}},
$$

where $V$ and $f$ stand for any electroweak gauge boson and SM fermion, respectively. The Higgs production cross section receives a suppression proportional to these same factors:

$$
\frac{\sigma(g g \rightarrow H)}{\sigma(g g \rightarrow H)_{\mathrm{SM}}}=R_{H f f}^{2}, \quad \frac{\sigma(q q \rightarrow q q H)}{\sigma(q q \rightarrow q q H)_{\mathrm{SM}}}(\mathrm{VBF})=\frac{\sigma(q q \rightarrow V H)}{\sigma(q q \rightarrow V H)_{\mathrm{SM}}}=R_{H V V}^{2}
$$

where VBF stands for "vector-boson fusion" and we have included the production processes relevant for the discussion in this article. The different decay widths scale with the corresponding couplings squared except for the $H \rightarrow \gamma \gamma$ channel that reads

$$
\Gamma(H \rightarrow \gamma \gamma)=\frac{\left(R_{H f f} I_{\gamma}+R_{H V V} J_{\gamma}\right)^{2}}{\left(I_{\gamma}+J_{\gamma}\right)^{2}} \Gamma^{\mathrm{SM}}(H \rightarrow \gamma \gamma),
$$

where

$$
I_{\gamma}=-\frac{8}{3} x_{t}\left[1+\left(1-x_{t}\right) f\left(x_{t}\right)\right], \quad J_{\gamma}=2+3 x_{W}\left[1-\left(2-x_{W}\right) f\left(x_{W}\right)\right]
$$


with $x_{t}=4 m_{t}^{2} / m_{H}^{2}, x_{W}=4 m_{W}^{2} / m_{H}^{2}$ and

$$
f(x)= \begin{cases}\arcsin [1 / \sqrt{x}]^{2}, & x \geq 1, \\ -\frac{1}{4}\left[\log \frac{1+\sqrt{1-x}}{1-\sqrt{1-x}}-\mathrm{i} \pi\right]^{2}, & x<1 .\end{cases}
$$

The $H \rightarrow \gamma Z$ is also modified in a similar way, with different loop functions. We do not give the explicit result as it will not be used in the following. ${ }^{4}$ The above equations completely determine all the relevant properties of the Higgs due to its pseudo-Goldstone nature (parameterized by the coefficient $\xi$ ). The Higgs becomes SM-like in the limit $\xi=0$. As an example, we show in Fig. 1 the Higgs production cross section times branching ratio

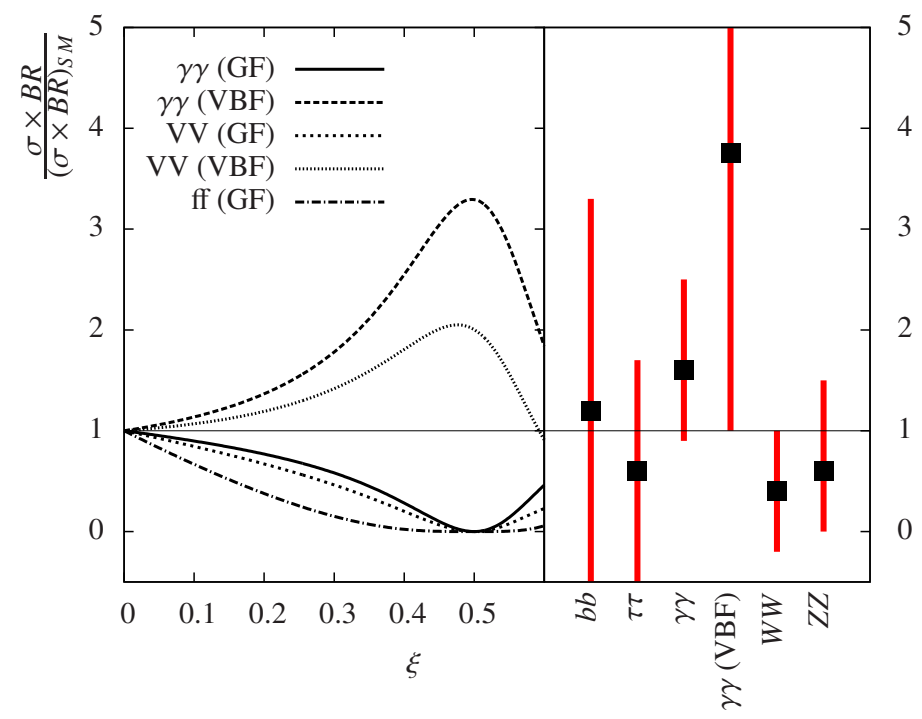

Figure 1. Left panel: Higgs production cross section times branching ratio into different channels in units of the corresponding SM process (Higgs production has been separated in gluon fusion and vector boson fusion, denoted by GF and VBF, respectively). Right panel: best fit value for the same observable obtained by the CMS collaboration [38, 39]. Both plots are for $m_{H}=125 \mathrm{GeV}$.

into different channels in units of the corresponding SM cross section as a function of $\xi$ for the case of a Higgs mass $m_{H}=125 \mathrm{GeV}$. For comparison we also show the best fit values for these cross sections as recently reported by CMS.

Once we have discussed the effects of Higgs compositeness on standard Higgs searches we can turn to the ingredients present in our new production mechanism. Since most likely only the first level of resonances will be accessible at the LHC, we use the two-site version of the $\mathrm{MCHM}_{5}[17,21]$ to study its LHC implications. ${ }^{5}$ All the details of the model can be

\footnotetext{
${ }^{4}$ The $H \rightarrow \gamma \gamma$ has this simple form because of cancellations due to the pseudo-Goldstone nature of the Higgs and the fact that a unique flavor structure is present in the model. See [34] for a detailed discussion and [35-37] for other cases.

${ }^{5}$ Strictly speaking, we use the deconstruction only for the collider search implications. As we have discussed, the features derived from the pseudo-Goldstone nature of the Higgs, which could be obtained in proper deconstructions of composite Higgs models [40, 41], have been taken directly from the holographic model in [33].
} 
found in the original reference [17]. Here we will just describe the features that are directly relevant for the Higgs production mechanism we want to study, namely the new massive gluons and vector-like quarks present in the spectrum, together with their couplings. The relevant new vector-like quarks are, for each family, two electroweak doublets $Q_{1 / 6}$ and $Q_{7 / 6}$ of hypercharges $1 / 6$ and $7 / 6$, respectively and one singlet, $\tilde{T}$, of hypercharge $2 / 3$

$$
Q_{1 / 6}^{(i)}=\left(\begin{array}{c}
T^{(i)} \\
B^{(i)}
\end{array}\right), \quad Q_{7 / 6}^{(i)}=\left(\begin{array}{c}
T_{5 / 3}^{(i)} \\
T_{2 / 3}^{(i)}
\end{array}\right), \quad \tilde{T}^{(i)},
$$

with masses $M_{Q_{1 / 6}^{(i)}}, M_{Q_{7 / 6}^{(i)}}, M_{T^{(i)}}$. There is also a massive gluon in the spectrum, a color octet vector boson denoted by $G$, with mass $M_{G}$.

The couplings of all the different particles are fixed by the couplings in the composite sector and the degree of compositeness of the different fields. The relevant couplings in the composite sector are the Yukawa couplings of the up and down sectors, that we take equal for simplicity, and the composite coupling of the heavy gluons

$$
Y_{*} \equiv Y_{* U}=Y_{* D}, \quad g_{* 3} .
$$

The degree of compositeness of each SM field can be parameterized by a mixing angle, denoting the degree of compositeness of the SM gluon $\left(\theta_{3}\right)$, left-handed $(\mathrm{LH})$ doublets $\left(\phi_{q}^{(i)}\right)$, charge $2 / 3$ singlets $\left(\phi_{u}^{(i)}\right)$ and charge $-1 / 3$ singlets $\left(\phi_{d}^{(i)}\right)$. Finally, in the $\mathrm{MCHM}_{5}$ model, there is an extra parameter describing the way the LH doublet is split between two sectors, denoted by the (small) angle $\phi_{2}^{(i)}$. Of course, not all of these parameters are independent. For instance the mass of the $Q_{1 / 6}$ is fixed in terms of the mass of $Q_{7 / 6}$ and the compositeness of the LH doublets

$$
M_{Q_{1 / 6}^{(i)}}=M_{Q_{7 / 6}^{(i)}} / \cos \phi_{q}^{(i)} .
$$

Similarly we have

$$
g_{3}=g_{* 3} \sin \theta_{3},
$$

with $g_{3}$ the SM QCD coupling, and

$$
m_{u^{(i)}} \approx \frac{v}{\sqrt{2}} Y_{* U} \sin \phi_{q}^{(i)} \sin \phi_{u}^{(i)}, \quad m_{d^{(i)}} \approx \frac{v}{\sqrt{2}} Y_{* D} \sin \phi_{2}^{(i)} \sin \phi_{d}^{(i)},
$$

where $m_{u, d^{(i)}}$ are the corresponding SM quark masses. Thus, we can use as independent parameters

$$
g_{* 3}, Y_{*}, M_{Q_{7 / 6}^{(i)}}, M_{\tilde{T}^{(i)}}, \sin \phi_{u}^{(i)} \equiv s_{u}^{(i)}, \text { and } \sin \phi_{2}^{(i)} \equiv s_{2}^{(i)},
$$

and compute all the other parameters in terms of these from the equations above.

The coupling of the massive gluon $G$ to the SM fermions is given by

$$
g_{G \psi \psi}=g_{3}\left(\sin ^{2} \phi_{\psi} \cot \theta_{3}-\cos ^{2} \phi_{\psi} \tan \theta_{3}\right),
$$

where $\phi_{\psi}$ is one of the $\phi_{q}^{(i)}, \phi_{u}^{(i)}$ or $\phi_{d}^{(i)}$, depending on the SM fermion involved. The couplings of the heavy gluon to one SM fermion and one composite resonance are given by

$$
g_{G \psi \Psi}=g_{3} \frac{\sin \phi_{\psi} \cos \phi_{\psi}}{\sin \theta_{3} \cos \theta_{3}},
$$


where the relevant combinations of $\psi \Psi$ are $u_{L}^{(i)} T_{L}^{(i)}, d_{L}^{(i)} B_{L}^{(i)}, u_{R}^{(i)} \tilde{T}_{R}^{(i)} \cdot{ }^{6}$ Finally, the coupling to two massive resonances has the form

$$
g_{G \Psi \Psi}=g_{3}\left(\cos ^{2} \phi_{\Psi} \cot \theta_{3}-\sin ^{2} \phi_{\Psi} \tan \theta_{3}\right),
$$

where $\phi_{\Psi}=\phi_{q}, \phi_{q}, \phi_{u}$ for $Q=T, B, \tilde{T}$, respectively. ${ }^{7}$

If the composite sector is strongly coupled, $g_{* 3} \gg 1$, we have $\cot \theta_{3} \gg 1$ and the heavy resonances are strongly coupled to the heavy gluon (except for maximally composite SM fermions). This large coupling and the large multiplicity (as mentioned in footnote 7 , there is a number of other massive resonances with coupling $\left.g_{3} \cot \theta_{3}\right)$ imply a very large contribution to the heavy gluon width. For large values of the mass the width is of the order of the mass itself and talking about resonances stops making sense. We show in
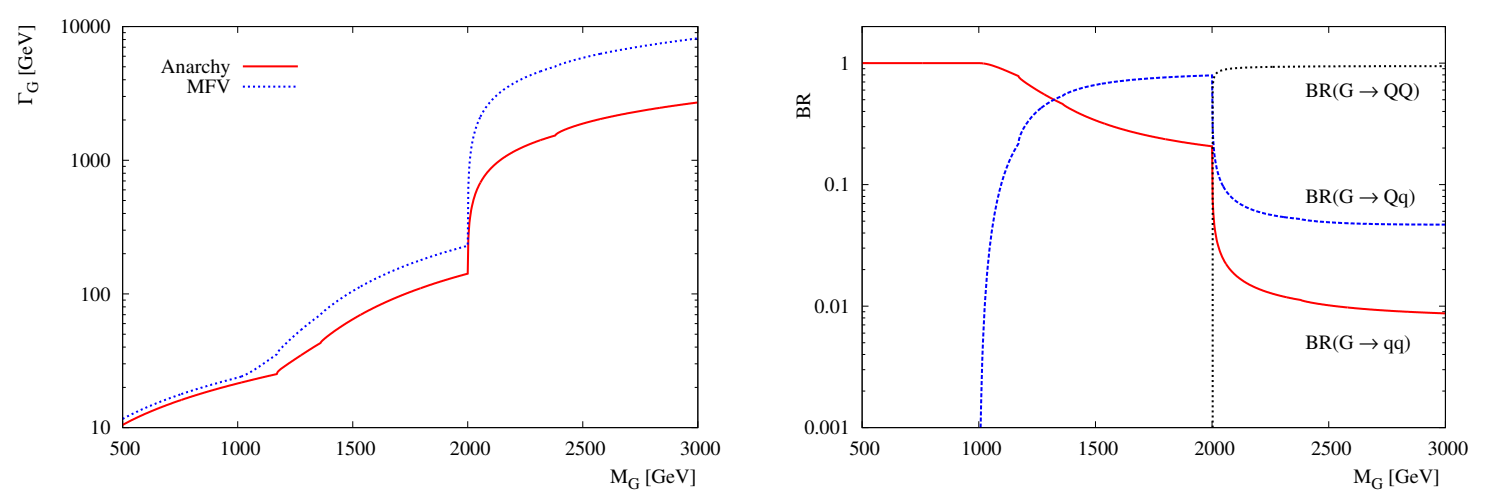

Figure 2. Left panel: massive gluon width as a function of its mass for the anarchic and MFV scenarios with $M_{F}=1 \mathrm{TeV}, s_{u}=0.6, s_{2}=0.1$ and $g_{3 *}=Y_{*}=3$ (in the anarchy scenario these values correspond to third generation quarks only, see text for details). Right panel: massive gluon branching fraction in two SM quarks (labeled $q q$ ), one SM and one heavy quark $(Q q)$ and two heavy quarks $(Q Q)$, respectively, for the MFV scenario.

Fig. 2 the massive gluon width (left panel) and branching ratios (right panel) as a function of its mass for $M_{Q_{7 / 6}}=M_{\tilde{T}}=1 \mathrm{TeV}, s_{u}=0.6, s_{2}=0.1$ and $g_{* 3}=Y_{*}=3$. In the left panel we have chosen two benchmark realizations of flavor. The first one corresponds to the standard anarchy scenario

$$
\text { Anarchy }\left(s_{u}^{(1)} \ll s_{u}^{(2)} \ll s_{u}^{(3)} \approx 1, \quad M^{(1,2)} \gg M^{(3)}\right),
$$

whereas the second one is the Minimal Flavor Violation (MFV) of [23] with mostly $u_{R}$ compositeness (see also [42-44] for alternatives)

$$
\operatorname{MFV} \quad\left(s_{u}^{(1)}=s_{u}^{(2)}=s_{u}^{(3)} \approx 1, \quad M^{(1,2)}=M^{(3)}\right) .
$$

\footnotetext{
${ }^{6} \mathrm{~A}$ charge $-1 / 3$ electroweak singlet can be also produced in association with $d_{R}^{(i)}$ but this process is not relevant for our Higgs production mechanism in the region of parameter space we are interested in.

${ }^{7}$ Other quarks present in the spectrum couple in pairs to $G$ with a similar structure but different values of the couplings. These couplings are nevertheless irrelevant for the process we are interested in, see Ref. [17] for details.
} 
where in both cases $M$ denote generically the fermion resonance masses. (Strictly speaking, in the MFV scenario only the $M_{\tilde{T}}$ masses have to be all equal. For simplicity we have assumed all of them to be family independent.) Due to the extreme widths developed when the decays into two massive fermionic resonances open up, we restrict ourselves to the region of parameter space in which these decay modes are kinematically suppressed (see [45] for an analysis of the case in which these decays are allowed). Thus, in the following we fix

$$
M_{F} \equiv M_{Q_{7 / 6}}=M_{\tilde{T}}=M_{G} / 2 .
$$

Also, although we will study the new Higgs production mechanism as a function of the different input parameters, we will often report results for a benchmark model, defined as

$$
\text { Benchmark Model: } M_{F}=M_{G} / 2, s_{u}=0.6, g_{* 3}=Y_{*}=3, s_{2}=0.1 \text {, }
$$

where the different coefficients refer to just the third generation in the anarchic case and to all three generations in the MFV scenario. Naturalness arguments and the recent hints for a light Higgs might prefer lighter fermion resonances for the third generation [46-48] (see also [49]). In these cases, if the heavy gluons are present, their width can easily exceed the perturbative limit. For example if we fix $M_{G}=3 \mathrm{TeV}, s_{u}=0.6, s_{2}=0.1$ and $g_{* 3}=Y_{*}=3$ in the anarchic scenario we get $\Gamma_{G} \gtrsim 0.9 M_{G}$ for $M_{F} \lesssim 1 \mathrm{TeV}$.

\section{Experimental Constraints}

Let us discuss current constraints on the model under consideration. Direct searches of new states impose only mild constraints on the parameter space allowed by electroweak precision tests. Nevertheless, the increasing precision of the experimental searches can have some impact on the parameter space as we describe here. There are four main types of searches with implications in our model. The first one is current Higgs searches, that are already starting to constrain the parameter space of composite Higgs models. The second one is searches for single production of new vector-like quarks that couple strongly to first generation SM quarks. The last two involve searches for new particles in $t \bar{t}$ and dijet final states, respectively. Processes leading to four-top final states represent a complementary probe of these models [50-56], but they are not sensitive to the range of masses we are considering here $[57,58]$.

\subsection{Higgs searches}

The implications of current Higgs searches on composite Higgs models have been studied in detail in $[7,8]$ and in more general extensions in [9-11, 59] (see also [12]). The result is that, for $m_{H}=125 \mathrm{GeV}$, the region

$$
0 \leq \xi \lesssim 0.4,
$$

is allowed. Other masses are also allowed for certain values of the degree of compositeness (for instance $m_{H} \approx 130 \mathrm{GeV}$ is allowed for $\xi \gtrsim 0.2-0.3$ ). We will use as benchmark values $m_{H}=125 \mathrm{GeV}$ and $\xi=0.2$ in this article but will also consider the effect of variations in these parameters. 


\subsection{Vector-like quark searches}

Our assumption on the masses of the fermion resonances, Eq. (2.18), was imposed to avoid too large a width for the massive gluon. Electroweak precision tests already impose stringent bounds on the masses of the heavy gluons (assuming that their mass is similar to the one of electroweak vector resonances), making the fermion resonances heavier than the reach of current searches. The only exception occurs in the case of fermion resonances that mix strongly with first generation quarks, as happens in the MFV scenario we are considering. It was noted in Refs. [60, 61] (see also [62] for lepton resonances) that dedicated searches at hadron colliders of electroweak single production of these fermion resonances could probe quite large masses. The main reason is the large cross section due to the presence of valence quarks in the initial state and the distinctive kinematics. In fact, ATLAS has recently performed such a search on their data [63] and as we discuss now, their null results imply the most stringent constraints for the MFV scenario in a large fraction of parameter space.

Ref. [63] reports $95 \%$ CL limits on the coupling $\tilde{\kappa}_{u U, u D}$ defined from the general parameterization of the gauge couplings

$$
\frac{g}{2 c_{W}} \tilde{\kappa}_{u U} \frac{v}{m_{U}} Z_{\mu} \bar{u}_{R} \gamma^{\mu} U_{R}+\frac{g}{\sqrt{2}} \tilde{\kappa}_{u D} \frac{v}{m_{D}} W_{\mu}^{+} \bar{u}_{R} \gamma^{\mu} D_{R}+\text { h.c. }
$$

where $g$ is the $S U(2)_{L}$ coupling, $c_{W}$ the cosine of the weak angle and $m_{U, D}$ are the masses of new vector-like quarks, $U$ and $D$, of charges $2 / 3$ and $-1 / 3$, respectively. The bounds on $\tilde{\kappa}^{2}$ are reported assuming only one type of quark at each time. This applies directly to our model in the case of the neutral current channel $\left(\tilde{\kappa}_{u U}\right)$. In the charged current channel, however, we have two new vector-like quarks, $B^{(1)}$ and $T_{5 / 3}^{(1)}$ in the notation of Eq. (2.7), instead of just one and the charge $5 / 3$ one has a production cross-section (which is proportional to $\tilde{\kappa}^{2}$ ) approximately two times the one of the charge $-1 / 3$ quark [61]. The net result is that the bounds from Ref. [63] on $\tilde{\kappa}_{u D}^{2}$ are in practice a factor of 3 stronger when interpreted in our model.

The value of $\tilde{\kappa}_{u U, u D}$ can be easily computed in our model, following the method outlined in section 13 of [64]. It is mostly sensitive to $s_{u}^{(1)}$ and $Y_{* U}$ although the dependence on the latter is milder. As an example, we show the value obtained in the MFV scenario with $Y_{* U}=3$ and $M_{F}=1 \mathrm{TeV}$, as a function of $s_{u}^{(1)}$ in Fig. 3, together with the corresponding experimental bounds as interpreted in our model. We see that for these values of the input parameters, only a relatively small degree of compositeness $s_{u}^{(1)} \lesssim 0.4$ is allowed. Due to limited statistics, only fermion masses up to $1 \mathrm{TeV}$, could be constrained in [63]. Thus, anything above that is currently experimentally allowed but we see from the figure that updates on these searches are likely to be one of the strongest constraints on the degree of compositeness of the $u_{R}$ quark in the MFV scenario.

\section{$3.3 t \bar{t}$ resonance searches}

In models of strong EWSB with a composite top, new vector resonances and in particular heavy gluons decay most of the time in $t \bar{t}$ pairs, which has been traditionally considered the golden discovery mode of such new particles. If the top is not fully composite, if other 


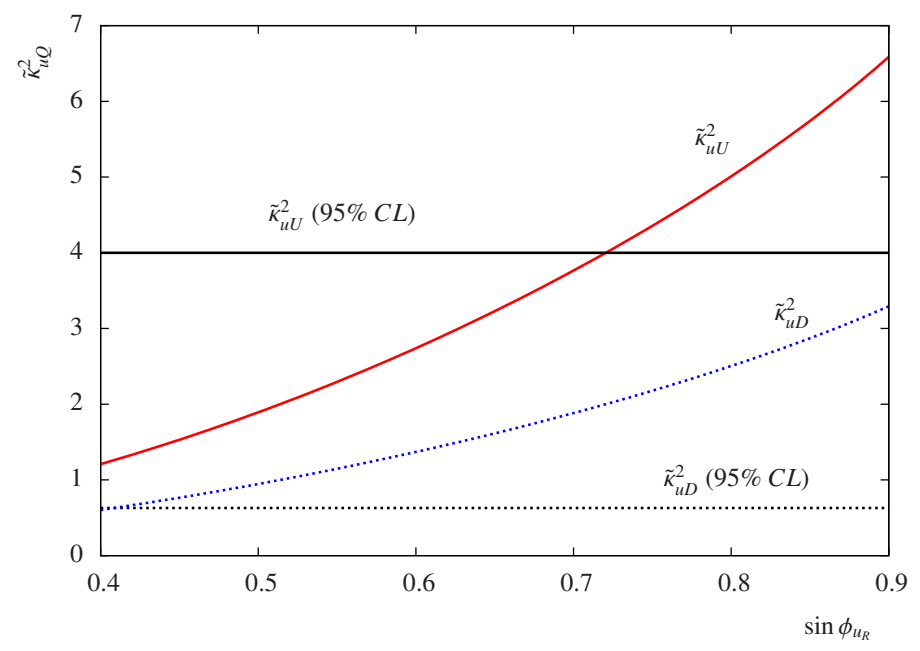

Figure 3. Values of $\tilde{\kappa}_{u U, u D}^{2}$ obtained in the MFV scenario with $Y_{* U}=3$ and $M_{F}=1$. The horizontal lines correspond to the $95 \%$ CL experimental upper bounds as interpreted in our model.

quarks can be as composite as the top (as happens in the MFV scenario), or if new decay channels involving fermion composite states are open, the branching fraction into $t \bar{t}$ can substantially change. As an example for the benchmark model in Eq.(2.19) with MFV we have $B R(G \rightarrow t \bar{t})=0.036$ and the $t \bar{t}$ production is at least an order of magnitude below current limits [65-67]. We will include the constraints resulting from these searches in our general analysis in section 5 .

\subsection{Di-jet searches}

We are considering models that depart from the standard composite models in two main aspects. The first is that we do not necessarily consider a completely composite $t_{R}$ [68]. The second is that we also consider the MFV scenario in which the $u_{R}$ and $c_{R}$ are as composite as the $t_{R}$. In this case, dijet production can impose stringent constraints in the model $[23,69]$. A very detailed study of the implication of dijet searches on contact interactions has been recently reported in [70]. Their original analysis, which considers the early LHC data of Ref. [71] with an integrated luminosity of just $36 \mathrm{pb}^{-1}$, has been updated to include the latest experimental results [72] with an integrated luminosity of 2.2 $\mathrm{fb}^{-1} .{ }^{8}$ Denoting the coupling of the first generation SM quarks to the massive gluon by

$$
G_{\mu}^{A}\left[g_{q_{L}} \bar{q}_{L} \gamma^{\mu} T^{A} q_{L}+g_{u_{R}} \bar{u}_{R} \gamma^{\mu} T^{A} u_{R}+g_{d_{R}} \bar{d}_{R} \gamma^{\mu} T^{A} d_{R}\right]
$$

we get the following effective Lagrangian after integration of the massive gluon, in the basis of $[70]$

$$
\mathcal{L}=\frac{c_{u u}^{(1)}}{M^{2}} \mathcal{O}_{u u}^{(1)}+\frac{c_{d d}^{(1)}}{M^{2}} \mathcal{O}_{d d}^{(1)}+\frac{c_{u d}^{(8)}}{M^{2}} \mathcal{O}_{u d}^{(8)}+\frac{c_{q q}^{(8)}}{M^{2}} \mathcal{O}_{q q}^{(8)}+\frac{c_{q u}^{(8)}}{M^{2}} \mathcal{O}_{q u}^{(8)}+\frac{c_{q d}^{(8)}}{M^{2}} \mathcal{O}_{q d}^{(8)}
$$

\footnotetext{
${ }^{8}$ We would like to thank O. Domenech, A. Pomarol and J. Serra for providing us with the updated analysis and for useful discussions.
} 
where the different coefficients read

$$
\begin{aligned}
& c_{u u}^{(1)}=-\frac{g_{u_{R}}^{2}}{6}, \quad c_{d d}^{(1)}=-\frac{g_{d_{R}}^{2}}{6}, \quad c_{u d}^{(8)}=-g_{u_{R}} g_{d_{R}}, \\
& c_{q q}^{(8)}=-\frac{g_{q_{L}}^{2}}{2}, \quad c_{q u}^{(8)}=-g_{q_{L}} g_{u_{R}}, \quad c_{q d}^{(8)}=-g_{q_{L}} g_{d_{R}} .
\end{aligned}
$$

The results of [70] can be directly applied to these coefficients to obtain the corresponding bound on $M_{G}$.

Direct dijet resonance searches can also constrain our model. We have simulated dijet signals in our model and compared the results after cuts with the bounds on simplified gaussian resonances reported in [73]. The corresponding limits are included in our final results.

\section{Searches for New Composite Higgs Production Mechanisms}

The new Higgs production mechanism we want to study consists of single production of new vector-like quarks (together with a SM quark) mediated by the exchange of a heavy color octet vector boson. The vector-like quark then decays into the composite Higgs and a SM quark. Sample diagrams of this production mechanism are shown in Fig. 4. The t-channel diagram on the right panel of the figure is only relevant for the MFV scenario in which first generation SM quarks are strongly composite. The final state is either $H t \bar{t}$ or $H j j$ (the
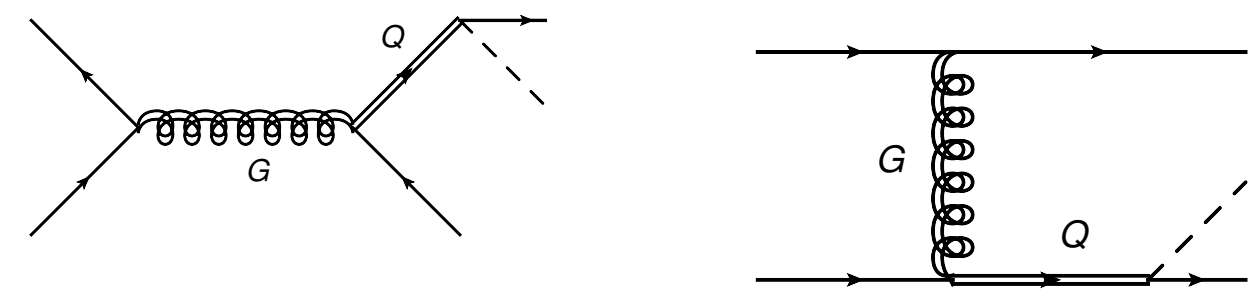

Figure 4. Sample diagrams for the new Higgs production mechanism. The t-channel exchange on the right is relevant for composite $u$ or $d$ quarks.

latter gives a substantial contribution only in the MFV scenario). The corresponding cross sections depend on the coupling of $G$ to the SM quarks, to $q Q$ and also on the branching fraction of the heavy quarks into a SM quark and the Higgs. The relevant such branching fractions are, in the limit of large masses [17]

$$
\operatorname{BR}\left(T^{(i)} \rightarrow u^{(i)} H\right) \approx 0.5, \quad \operatorname{BR}\left(\tilde{T}^{(i)} \rightarrow u^{(i)} H\right) \approx 0.25 .
$$

Other channels either do not result in a Higgs or their production is strongly suppressed due to small degree of compositeness. We show in Fig. 5 the production cross section times branching ratio for the $H t \bar{t}$ (left) and $H j j$ (right) channels in the benchmark model of Eq.(2.19) within the MFV scenario. In the anarchic scenario the $H j j$ channel is negligible and the $H t \bar{t}$ is enhanced by a factor $\approx 25-40 \%$, depending on the value of $M_{G}$. 

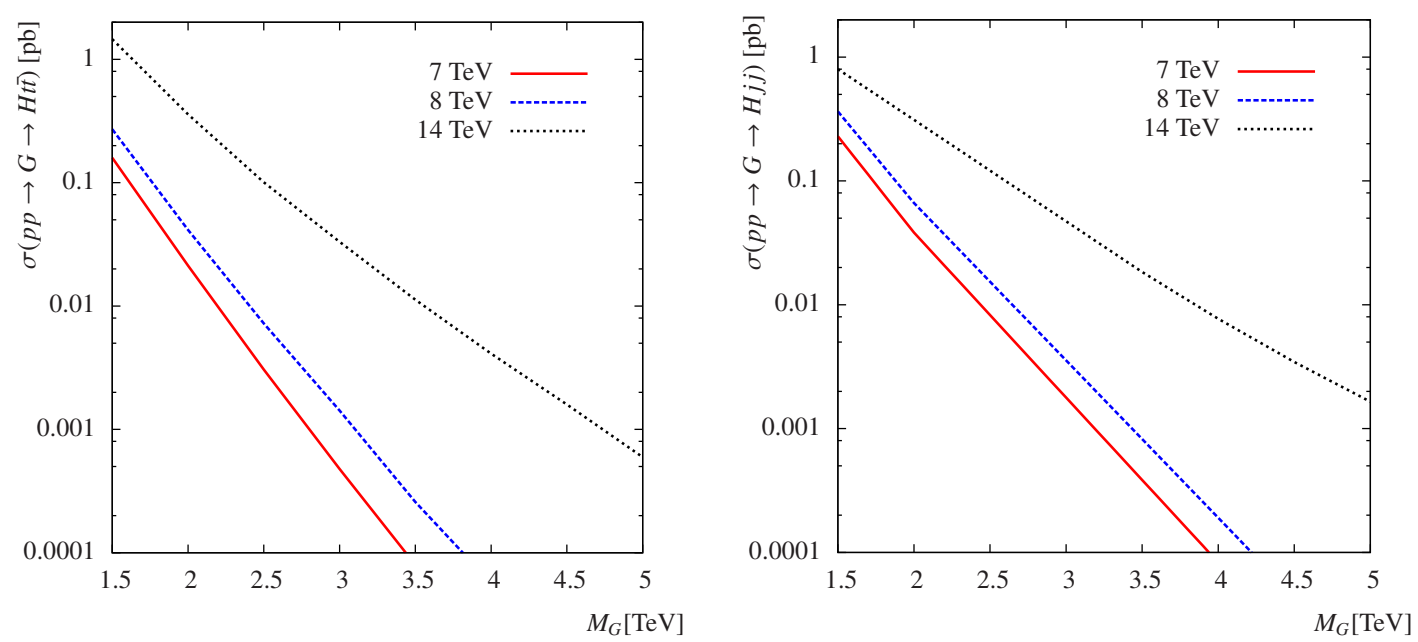

Figure 5. Left panel: $H t \bar{t}$ production cross section in the benchmark MFV composite model mediated by a color octet vector resonance with decay into a fermionic resonance and a top quark. Right panel: same for the $H j j$ channel.

In the following we describe dedicated analyses for the $H t \bar{t}$ and $H j j$ channels. We have considered three different configurations for the LHC parameters, namely $5 \mathrm{fb}^{-1}$ integrated luminosity at $\sqrt{s}=7 \mathrm{TeV}, 20 \mathrm{fb}^{-1}$ integrated luminosity at $\sqrt{s}=8 \mathrm{TeV}$ and $100 \mathrm{fb}^{-1}$ integrated luminosity at $\sqrt{s}=14 \mathrm{TeV}$. The range of masses probed with the first two configurations (that we call the low energy phase) is quite different from the one probed by the high energy phase (the third option). Thus, the analyses are also different depending on the phase. In particular, as we describe below, the analysis of the $H t \bar{t}$ channel in the high energy phase benefits from using boosted techniques.

We have used MADGRAPH V4.5.0 [74] and ALPGEN V.2.13 [75] to generate signal and background partonic events. Such events have then been passed through PYTHIA 6.4 [76] for hadronization and showering and DELPHES V1.9 [77] for detector simulation. Regarding the latter we have used a tuned version of the standard ATLAS card that results in an very good agreement with published experimental results. We have used the CTEQ6L1 PDFs and the default values of the renormalization and factorization scales. The backgrounds have been matched using the MLM method. In our analyses we define jets with a cone size $\Delta R=0.7, p_{T}(j)>30 \mathrm{GeV}$ and $\left|\eta_{j}\right|<5$. Isolated charged leptons $(e$ or $\mu)$ are considered when $p_{T}(l)>20 \mathrm{GeV}$ and $\left|\eta_{l}\right|<2.5$. We have assumed a b-tagging efficiency of 0.7 in our analyses. Finally, we use as discriminating variable

$$
S_{T} \equiv \sum_{j=1}^{n_{j}} p_{T}(j)+\sum_{l=1}^{n_{l}} p_{T}(l)+E_{T},
$$

where $n_{j, l}$ is the relevant number of jets or leptons (ordered according to their $p_{T}$ ), which depends on the analysis and will be specified below. We list in Table 1 the main backgrounds and two sample points in parameter space for our benchmark model together with their cross sections. 


\begin{tabular}{|l|c|c|c|}
\hline Process & LHC7 & LHC8 & LHC14 \\
& $\sigma[\mathrm{pb}]$ & $\sigma[\mathrm{pb}]$ & $\sigma[\mathrm{pb}]$ \\
\hline$H t \bar{t}\left(M_{G}=2 \mathrm{TeV}, \mathrm{MFV}\right)$ & 0.0213 & 0.0414 & 0.270 \\
$H t \bar{t}\left(M_{G}=3 \mathrm{TeV}, \mathrm{MFV}\right)$ & 0.00053 & 0.0015 & 0.033 \\
$H t \bar{t}\left(M_{G}=2 \mathrm{TeV}\right.$, Anarchy) & 0.029 & 0.058 & 0.39 \\
$H t \bar{t}\left(M_{G}=3 \mathrm{TeV}\right.$, Anarchy) & 0.00061 & 0.0018 & 0.046 \\
$H j j\left(M_{G}=2 \mathrm{TeV}, \mathrm{MFV}\right)$ & 0.04 & 0.07 & 0.44 \\
\hline$t \bar{t}+0-4$ jets (semileptonic+leptonic) & 47.9 & 70.47 & 268.55 \\
$t \bar{t} b \bar{b}$ & 0.09 & 0.15 & 0.85 \\
$Z+1-4$ jets (leptonic) & 530.5 & 641 & 1423 \\
$W W+0-2$ jets (semileptonic+leptonic) & 15 & 22.6 & 49 \\
$W+1-2$ jets (p $>150$ GeV, leptonic $)$ & - & - & 84.9 \\
$W+1-4$ jets (leptonic) & 5133 & 6489 & - \\
\hline
\end{tabular}

Table 1. Cross section for different the signal and main backgrounds for different values of the LHC energy. In $t \bar{t}$ and $t \bar{t} b \bar{b}$ leptonic decays refer to $e$ or $\mu$, in the other cases decays into $\tau$ are also included. The corresponding branching fractions are included in the calculation of the cross section (when the decays -leptonic or semileptonic- are explicitly stated).

Given a number of signal $(s)$ and background $(b)$ events after the corresponding cuts, we compute the statistical significance of the signal from

$$
\mathcal{S}(s, b)=\sqrt{2 \times\left[(s+b) \ln \left(1+\frac{s}{b}\right)-s\right]} .
$$

We describe in this section our suggested analyses and the effect they have on signal and background for a specific point in parameter space. We will then present our results in the next section as a function of the input parameters.

\section{1 $H t \bar{t}$ analysis: low energy phase}

The mass range that can be probed at the LHC within the low energy phase $(\sqrt{s}=7$ or $8 \mathrm{TeV}$ ) is relatively low. This means that the decay products are not extremelly boosted. We have found that traditional analyses are more efficient probing this region of parameter space than analysis that use boosted techniques. Also, since we have the leptonic top decays to trigger on, we can afford to use the main Higgs decay channel, namely $b \bar{b}$, with a branching ratio $\mathrm{BR}(H \rightarrow b \bar{b})=0.48$ for the benchmark model in Eq. (2.19) (the changes in the BR for different values of the input parameters can be recovered using the equations in Section 2 and are shown, for reference, in Fig. 6). We are therefore interested in the following process

$$
p p \rightarrow G \rightarrow T \bar{t}+\bar{T} t \rightarrow H t \bar{t} \rightarrow 4 b+2 j+l+E_{T} .
$$

The main backgrounds are $t \bar{t}$ and $t \bar{t} b \bar{b}$. In order to reduce the number of background events to manageable values we impose the following initial cuts 
- At least 4 jets, of which at least 3 must be tagged as b-jets.

- At least 1 isolated charged lepton.

- A cut on $S_{T}$ (in this case we have $n_{j}=4$ and $n_{l}=1$ ) that depends on the test $M_{G}$ we are considering

$$
S_{T}>0.9,1.1,1.5 \mathrm{TeV} \text { for } M_{G}=1.5,2,2.5 \mathrm{TeV} \text {. }
$$

\begin{tabular}{|c|c|c|c|}
\hline \multicolumn{4}{|c|}{ sample $H t \bar{t}$ analysis (low energy phase) } \\
\hline cut & $\epsilon_{M_{G}=2 \mathrm{TeV}}$ & $\epsilon_{t \bar{t}}$ & $\epsilon_{t \bar{t} b \bar{b}}$ \\
\hline$n_{j} \geq 4$ & 77.31 & 52.16 & 91.85 \\
\hline$n_{l} \geq 1$ & 66.86 & 63.02 & 42.84 \\
\hline$n_{b} \geq 3$ & 35.31 & 2.64 & 33.08 \\
\hline$S_{T}$ & 75.01 & 0.12 & 1.20 \\
\hline Total & 13.69 & 0.00108 & 0.156 \\
\hline
\end{tabular}

\begin{tabular}{|c|c|c|c|}
\hline \multicolumn{4}{|c|}{$H t \bar{t}$ (low energy phase) } \\
\hline$M_{G}[\mathrm{TeV}]$ & $\epsilon_{s}$ & $\epsilon_{t \bar{t}}$ & $\epsilon_{t \bar{t} \bar{b}}$ \\
\hline 1.5 & 15.8 & 0.00652 & 0.514 \\
\hline 2.0 & 13.69 & 0.00108 & 0.156 \\
\hline 2.5 & 9.67 & 0.000292 & 0.0174 \\
\hline 3.0 & 9.14 & 0.000292 & 0.0174 \\
\hline
\end{tabular}

Table 2. Left panel: Cut by cut efficiencies for the signal and main backgrounds for the $H t \bar{t}$ analysis in the low energy phase for a sample point (benchmark model with MFV and $M_{G}=2$ $\mathrm{TeV}$ ). Right panel: global efficiencies for the signal and relevant backgrounds as a function of $M_{G}$. All efficiencies are reported as per cent.

We show in the left panel of Table 2, the efficiencies of the different cuts for the main backgrounds and our signal for the MFV realization of our benchmark model, Eq. (2.19), with $M_{G}=2 \mathrm{TeV}$. The global efficiencies for the signal (again BM with MFV) and relevant backgrounds are reported, as a function of $M_{G}$ in the right panel of the table. From the numbers in this table and the cross sections for the signal (times the $B R(H \rightarrow b \bar{b})=0.48$ for $m_{H}=125 \mathrm{GeV}$ and $\left.\xi=0.2\right)$ and background reported in Table 1 we obtain a statistical significance of

$$
\begin{aligned}
& \mathcal{S}(7.1,3.3)=3.1 \quad\left(\mathcal{L}=5 \mathrm{fb}^{-1}, \sqrt{s}=7 \mathrm{TeV}, M_{G}=2 \mathrm{TeV}, \mathrm{MFV}\right) \\
& \mathcal{S}(55.9,19.9)=10.8 \quad\left(\mathcal{L}=20 \mathrm{fb}^{-1}, \sqrt{s}=8 \mathrm{TeV}, M_{G}=2 \mathrm{TeV}, \mathrm{MFV}\right)
\end{aligned}
$$

In the case of $\sqrt{s}=8 \mathrm{TeV}$ a luminosity of just $4.3 \mathrm{fb}^{-1}$ would suffice for a $5 \sigma$ discovery. In the anarchy scenario, there is a $\approx 35 \%$ (38\%) enhancement for $\sqrt{s}=7$ (8) $\mathrm{TeV}$ of the signal, resulting in the following statistical significances

$$
\begin{aligned}
& \mathcal{S}(9.5,3.3)=4 . \quad\left(\mathcal{L}=5 \mathrm{fb}^{-1}, \sqrt{s}=7 \mathrm{TeV}, M_{G}=2 \mathrm{TeV}, \text { Anarchy }\right) \\
& \mathcal{S}(77.2,19.9)=13.9 \quad\left(\mathcal{L}=20 \mathrm{fb}^{-1}, \sqrt{s}=8 \mathrm{TeV}, M_{G}=2 \mathrm{TeV}, \text { Anarchy }\right) .
\end{aligned}
$$

As we will discuss in the next section, in which we describe our results as a function of the input parameters, current constraints from dijet contact interactions imply a bound $M_{G} \geq 2.5 \mathrm{TeV}$ for the benchmark model in the anarchy scenario. These values cannot be probed with the $7 \mathrm{TeV}$ run but with $\sqrt{s}=8 \mathrm{TeV}$ it should be possible to discover (exclude) it with $20(5) \mathrm{fb}^{-1}$. 


\subsection{HtT analysis: high energy phase}

In the high energy phase, $\sqrt{s}=14 \mathrm{TeV}$, larger masses can be probed. In this case the decay products of $G$ and $Q$ are highly boosted and one can benefit from the use of boosted techniques. In this study we use a very simple technique, based on fat jet invariant masses [7880]. Clearly there is room for improvement if more sophisticated tools are used [81, 82]. The new set of cuts optimized for the larger masses probed are the following

- At least 3 jets, with a minimum of $2 \mathrm{~b}$ tags.

- At least 1 isolated charged lepton.

- All jets are then ordered according to their invariant mass and the first two jets are required to have invariant masses close to the top and Higgs mass, respectively, $\left|m_{j_{1}}-m_{t}\right| \leq 40 \mathrm{GeV}$ and $\left|m_{j_{2}}-m_{H}\right| \leq 40 \mathrm{GeV}$ (here $j_{1,2}$ are the jets with the largest and second largest invariant masses).

- A cut on $S_{T}$ (in this case we have $n_{j}=3$ and $n_{l}=1$ ) that depends on the test $M_{G}$ we are considering

$$
S_{T}>1.2,1.5,1.7,2 \mathrm{TeV} \text { for } M_{G}=2,2.5,3, \geq 3.5 \mathrm{TeV} .
$$

\begin{tabular}{|c|c|c|c|}
\hline \multicolumn{4}{|c|}{ sample $H t \bar{t}$ analysis (high energy phase) } \\
\hline cut & $\epsilon_{M_{G}=3 \mathrm{TeV}}$ & $\epsilon_{t \bar{t}}$ & $\epsilon_{t \bar{t} b \bar{b}}$ \\
\hline$n_{j} \geq 3$ & 98.03 & 85.46 & 98.88 \\
\hline$n_{l} \geq 1$ & 75.24 & 61.08 & 45.16 \\
\hline$n_{b} \geq 2$ & 64.38 & 29.49 & 68.50 \\
\hline$m_{j_{1}} \sim m_{t}$ & 58.08 & 0.22 & 1.70 \\
\hline$m_{j_{2}} \sim m_{h}$ & 72.70 & 15.36 & 31.72 \\
\hline$S_{T}$ & 90.07 & 10.24 & 18.10 \\
\hline Total & 18.06 & 0.00054 & 0.0298 \\
\hline
\end{tabular}

\begin{tabular}{|c|c|c|c|}
\hline \multicolumn{4}{|c|}{$H t \bar{t}$ (high energy phase) } \\
\hline$M_{G}[\mathrm{TeV}]$ & $\epsilon_{s}$ & $\epsilon_{t \bar{t}}$ & $\epsilon_{t \bar{t} b \bar{b}}$ \\
\hline 2.0 & 11.74 & 0.00265 & 0.1021 \\
\hline 2.5 & 15.61 & 0.00095 & 0.0518 \\
\hline 3.0 & 18.06 & 0.00054 & 0.0298 \\
\hline 3.5 & 17.74 & 0.00027 & 0.0188 \\
\hline 4 & 19.08 & 0.00027 & 0.0188 \\
\hline 4.5 & 19.40 & 0.00027 & 0.0188 \\
\hline
\end{tabular}

Table 3. Left panel: Cut by cut efficiencies for the signal and main backgrounds for the $H t \bar{t}$ analysis in the high energy phase for a sample point (benchmark model with MFV and $M_{G}=3$ $\mathrm{TeV}$ ). Right panel: global efficiencies for the signal and relevant backgrounds as a function of $M_{G}$. All efficiencies are reported as per cent.

The results of these cuts on the main backgrounds and the signal are reported in table 3 . In the left panel we report cut-by-cut efficiencies for a sample signal point (MFV for the benchmark model with $M_{G}=3 \mathrm{TeV}$ ) whereas in the right panel we report the global efficiencies as a function of $M_{G}$. The corresponding statistical significance is

$$
\mathcal{S}(288,170)=18 \quad\left(\mathcal{L}=100 \mathrm{fb}^{-1}, \sqrt{s}=14 \mathrm{TeV}, M_{G}=3 \mathrm{TeV}, \mathrm{MFV}\right) .
$$

A $5 \sigma$ discovery could be reached with this energy using just an integrated luminosify of 7.5 $\mathrm{fb}^{-1}$. The $38 \%$ enhancement in the anarchy case results in

$$
\mathcal{S}(398,170)=24 \quad\left(\mathcal{L}=100 \mathrm{fb}^{-1}, \sqrt{s}=14 \mathrm{TeV}, M_{G}=3 \mathrm{TeV}, \text { Anarchy }\right) .
$$


Results for other regions of parameter space will be reported in the next section.

\subsection{Hjj analysis: high energy phase}

The last analysis we are going to describe is relevant for the MFV realization of flavor in which the SM quarks produced in association with the Higgs are first and second generation quarks. The Higgs is then produced in association with two jets with a cross section given, for our benchmark model, in Fig. 1 (right panel). Even if the two extra jets are quite hard or forward, depending on whether we have an $s-$ or $t$-channel contribution, the signal is completely swamped by backgrounds if we consider the $H \rightarrow b \bar{b}$ decay channel. We are therefore forced to consider the $H \rightarrow W W^{*}$ channel, with a $B R\left(H \rightarrow W W^{*}\right)=0.33$ for our benchmark model. Even so, the relatively small cross sections and the huge $W+j$ background makes the dilepton mode the only one in which the signal can realistically extracted from the background. The penalty to pay is then the low cross sections and we will be in all cases statistics limited. Due to this limitation, we only consider the high energy LHC phase for this channel. The process we are interested in is therefore

$$
p p \rightarrow G \rightarrow U \bar{u}+\bar{U} u \rightarrow H u \bar{u} \rightarrow 2 j+2 l+E_{T} .
$$

The main backgrounds are $W+$ jets, $Z+$ jets, $W W+$ jets and $t \bar{t}+$ jets. We have simulated them as described in Table 1 . In order to have enough statistics for the $W+$ jets sample we have generated it with up to hard 2 jets, $p_{T} \geq 150 \mathrm{GeV}$, fom matrix elements (all other jets have been generated by the parton shower). The cuts we propose are

- At least 2 and no more than 6 jets.

- Exactly 2 charged leptons, both with $p_{T}(l) \geq 50 \mathrm{GeV}$ and $\left|\Delta \phi\left(l_{1}, l_{2}\right)\right| \leq 0.5$.

- A veto on b-tagged jets (no jet should be tagged as a b-jet).

- $p_{T}\left(j_{1}\right)>400 \mathrm{GeV}, p_{T}\left(j_{2}\right)>200 \mathrm{GeV}\left(j_{1,2}\right.$ denote the two hardest jets).

- A cut on the invariant mass of the two charged leptons $15 \mathrm{GeV} \leq m_{l l} \leq 70 \mathrm{GeV}$.

- A cut on the transverse mass of the Higgs decay products $m_{T}\left(l, l, E_{T}\right)<120 \mathrm{GeV}$. Where the transverse mass is defined as

$$
m_{T}=\sqrt{\left(E_{T}^{l l}+E_{T}\right)^{2}-\left|\mathbf{p}_{T}^{l l}+\mathbf{p}_{T}\right|^{2}},
$$

with $E_{T}^{l l}=\sqrt{\left|\mathbf{p}_{T}^{l l}\right|^{2}+m_{l l}^{2}},\left|\mathbf{p}_{T}\right|=E_{T}$, and $\left|\mathbf{p}_{T}^{l l}\right|=p_{T}^{l l}$.

- The following cut on $S_{T}$ using $n_{j}$ the number of jets between 2 and 6 from the first cut and $n_{l}=2$ as a function of the test $M_{G}$

$$
S_{T}>1.5,2.1,2.3 \mathrm{TeV} \text { for } M_{G}=2,2.5, \geq 3 \mathrm{TeV}
$$




\begin{tabular}{|c|c|c|c|}
\hline Cut & $\epsilon_{M_{G}=2 \mathrm{TeV}}$ & $\epsilon_{W}$ & $\epsilon_{t \bar{t}}$ \\
\hline $2 \leq n_{j} \leq 6$ & 99 & 76 & 96 \\
$n_{l}=2, p_{T}(l) \geq 50 \mathrm{GeV},|\Delta \phi| \leq 0.5$ & 28 & 0.0206 & 0.158 \\
$n_{b}=0$ & 95 & 86 & 22 \\
$p_{T}\left(j_{1}\right)>400 \mathrm{GeV}, p_{T}\left(j_{2}\right)>200 \mathrm{GeV}$ & 79 & 31 & 4.8 \\
$15<m_{l l}<70 \mathrm{GeV}$ & 91 & 47 & 75 \\
$m_{T}$ (Higgs) $<120 \mathrm{GeV}$ & 62 & 19 & 17 \\
$S_{T}$ & 97 & 98 & 97 \\
\hline Total & 12 & $3.6 \times 10^{-4}$ & $2 \times 10^{-4}$ \\
\hline
\end{tabular}

Table 4. Cut by cut efficiencies for the signal (benchmark model with MFV and $M_{G}=2 \mathrm{TeV}$ ) and the main backgrounds $(W+$ jets and $t \bar{t})$ in the $H j j$ channel at $\sqrt{s}=14 \mathrm{TeV}$. All efficiencies are reported as per cent.

The effect of the cuts on the different backgrounds and our signal for the benchmark model, MFV realization, with $M_{G}=2 \mathrm{TeV}$ are described in Table 4 . The cuts completely kill the $Z$ and $W W$ backgrounds which are therefore not reported. The cut on $S_{T}$ has no effect for this mass but is relevant for heavier masses. The corresponding statistical significance, taking into account the $B R\left(H \rightarrow W W^{*} \rightarrow l \nu l \nu\right) \approx 0.015$, is

$$
\mathcal{S}(81,85)=7.8 \quad\left(\mathcal{L}=100 \mathrm{fb}^{-1}, \sqrt{s}=14 \mathrm{TeV}, M_{G}=2 \mathrm{TeV}, \mathrm{MFV}\right) .
$$

The global efficiencies for the signal and the main backgrounds as a function of the test mass are given in Table 5 .

\begin{tabular}{|c|c|c|c|c|}
\hline$M_{G}$ & $\epsilon_{M_{G}=2 \mathrm{TeV}}$ & $\epsilon_{W}$ & $\epsilon_{t \bar{t}}$ & $\epsilon_{W W}$ \\
\hline 1.5 & 10.4 & 0.00148 & 0.00096 & 0.01 \\
\hline 2 & 11.75 & 0.000361 & 0.0002 & 0 \\
\hline 2.5 & 6.82 & $7.4 \times 10^{-5}$ & $1.57 \times 10^{-5}$ & 0 \\
\hline 3 & 7.26 & $4.54 \times 10^{-5}$ & 0 & 0 \\
\hline 3.5 & 8.15 & $4.54 \times 10^{-5}$ & 0 & 0 \\
\hline
\end{tabular}

Table 5. Global efficiencies for the signal and main backgrounds as a function of the test mass in the $H j j$ channel at $\sqrt{s}=14 \mathrm{TeV}$. All efficiencies are reported as per cent.

\section{Results and Discussion}

The details of our analyses and the results for the benchmark model have been discussed in full detail in the previous section. We now proceed to report our results as a function of the most relevant input parameters. We have found that the discovery limits or exclusion bounds are not very sensitive to the composite Yukawa couplings $Y_{*}$. The main sensitivity is to the composite coupling of the heavy gluons, $g_{* 3}$, and the degree of compositeness of 
the $u_{R}$, parameterized by $s_{u}$. In the next three subsections we describe our results as a function of these parameters in the MFV scenario for the $H t \bar{t}$ and $H j j$ channels and in the anarchic scenario for the $H t \bar{t}$ channel, respectively. In all cases we show our results in the form of contour plots of the required luminosity for a $5 \sigma$ discovery, defined as $\mathcal{S}(s, b)=5$, see Eq. (4.3). We also show contours of the luminosity required for the expected $95 \%$ exclusion bound. We have computed this bound by requiring

$$
\mathrm{CL}_{s} \equiv \frac{\mathrm{CL}_{s+b}}{\mathrm{CL}_{b}} \leq 0.05, \quad\left[\mathrm{CL}_{x}=P\left(n \leq n_{\mathrm{obs}} \mid x\right)\right]
$$

with $P(n, x)$ the Poisson distribution and in order to set the expected bound we have fixed the observed number of events to $b$ (the number of background events). Finally, we also report current exclusion limits from other searches as discussed in Section 3.
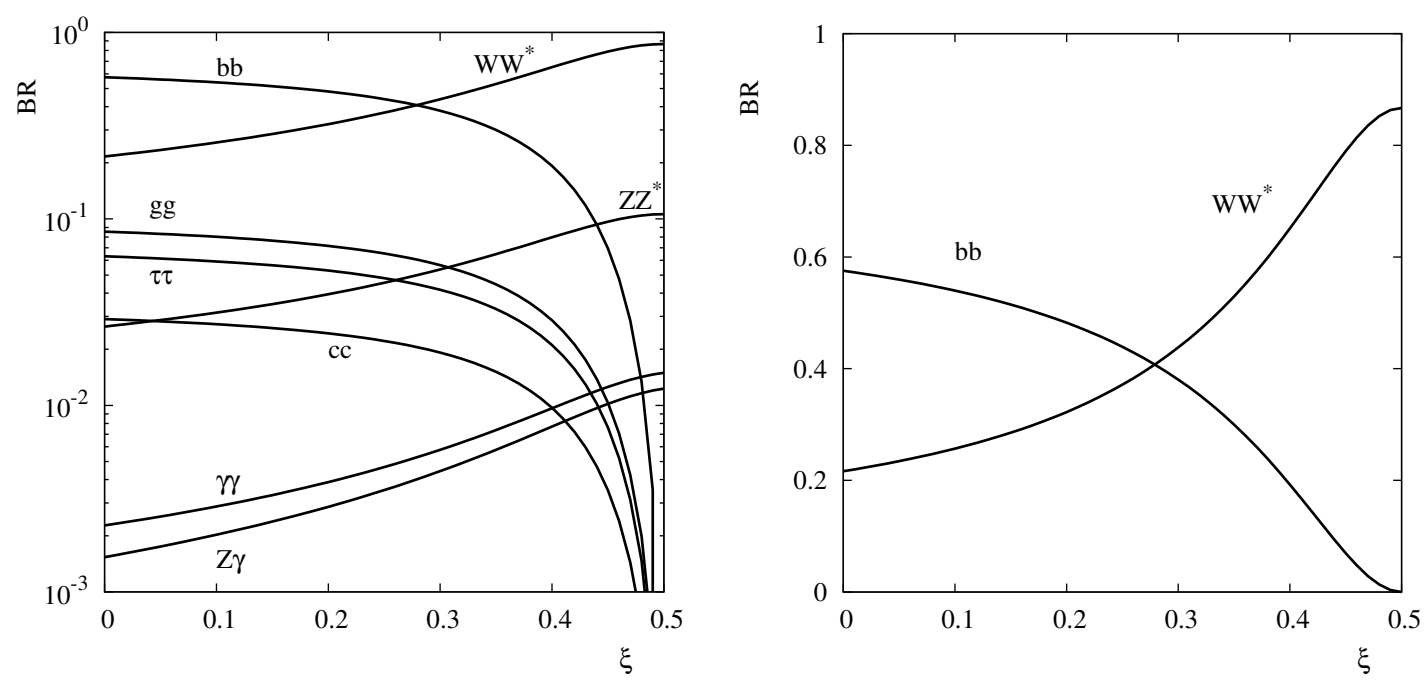

Figure 6. Branching ratios of a composite Higgs of mass $125 \mathrm{GeV}$ as a function of the degree of compositeness in logarithmic, left panel, and linear, right panel, scales (for the latter only the two channels used here are shown).

These results can be easily translated to different values of the degree of Higgs compositeness $(\xi)$. The number of background events for a fixed luminosity and center of mass energy can be obtained from the cross sections in Table 1 and the efficiencies in Tables 2-4. The number of signal events can then be inferred from the value of $\mathcal{S}(s, b)$ or $\mathrm{CL}_{s}$ in the figures in the next three subsections. This number of signal events can then be re-scaled by the ratio of Higgs branching ratio in the corresponding channel for the different values of $\xi$ and the new discovery reach or bound can be computed. To make this scaling easier we display in Fig. 6 the different Higgs branching ratios as a function of $\xi$.

\subsection{Ht $\bar{t}$ channel: MFV scenario}

Our main results for the $H t \bar{t}$ channel in the MFV scenario are shown in Fig. 7, as a function of $s_{u}$ and $M_{G}$ (left column) and as a function of $g_{* 3}$ and $M_{G}$ (right column). The three 

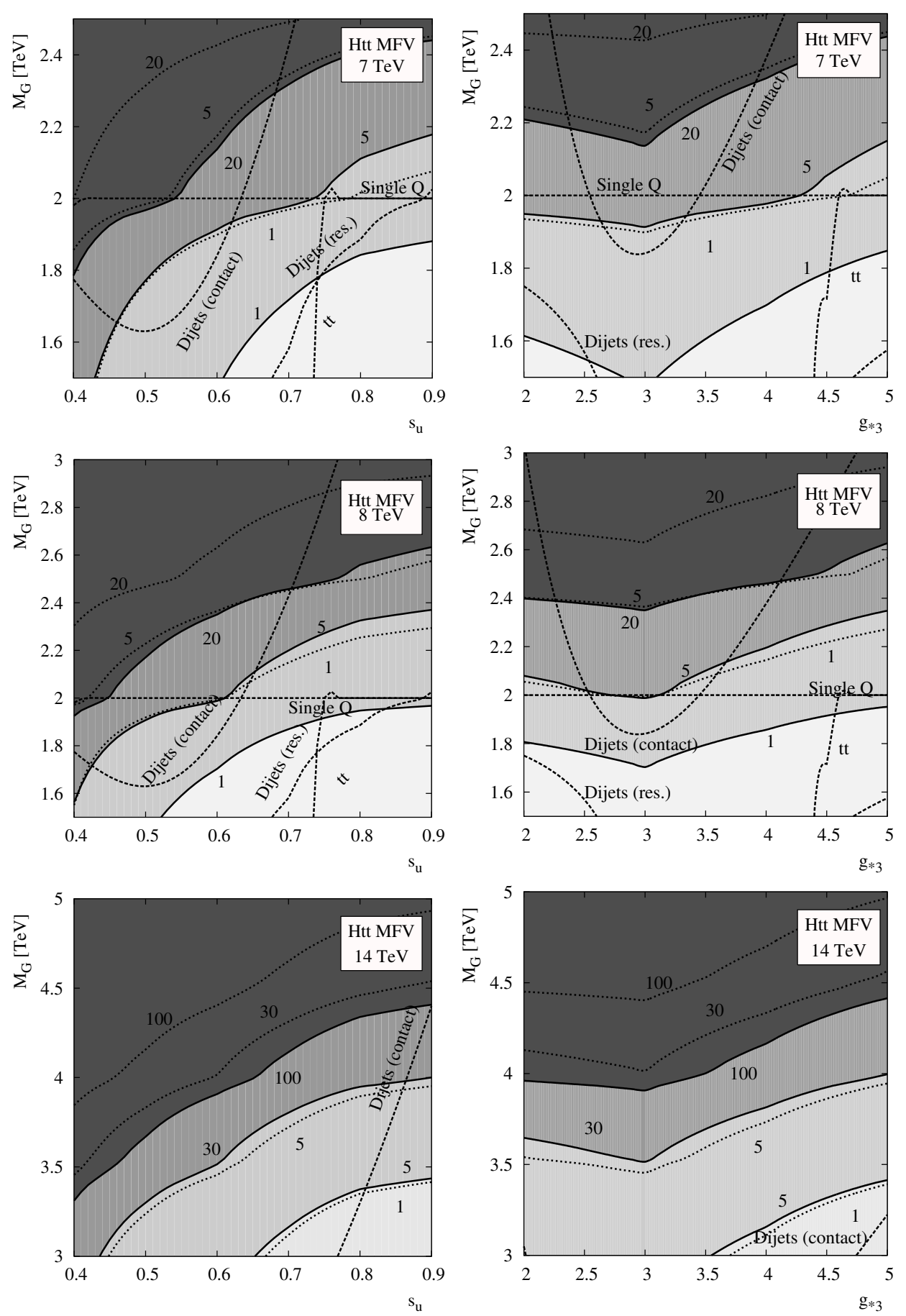

Figure 7. Contours of required luminosity for a $5 \sigma$ discovery (bands and solid lines) and $95 \%$ exclusion limits (dotted lines) as a function of $s_{u}$ and $M_{G}$ (left column) and $g_{* 3}$ and $M_{G}$ (right column) for $\sqrt{s}=7,8$ and $14 \mathrm{TeV}$ (first, second and third rows, respectively) in the $H t \bar{t}$ channel (MFV scenario). Current bounds are shown with dashed lines (the area below the dashed lines is excluded). 
rows of plots correspond, from top to bottom, to $\sqrt{s}=7,8$ and $14 \mathrm{TeV}$, respectively. The discovery (exclusion) contours are represented by solid (dotted) lines. Current constraints are represented with dashed lines. The excluded region is the area below the corresponding line. Single production searches [63] are quite restrictive, independently of the input parameters. Dijet limits on quark contact interactions (we have found that direct dijet resonance searches lead to weaker constraints) can be also very restrictive, particularly for large values of the $u_{R}$ degree of compositeness and for very small or very large values of $g_{* 3}$. The summary of our results for this channel is the following:

- Current constraints on the model would not allow for a $5 \sigma$ discovery with the 2011 data set at $\sqrt{s}=7 \mathrm{TeV}$. However, $95 \%$ exclusion bounds could be set in the region $M_{G} \sim 2-2.3 \mathrm{TeV}, s_{u} \sim 0.5-0.7$ and $g_{* 3} \sim 2.5-4$ within the currently allowed region of parameter space.

- Considering now the $2012 \sqrt{s}=8 \mathrm{TeV}$ run, a much larger region of the parameter space currently allowed by experimental data can be explored. For example $M_{G} \sim 2.5$ $\mathrm{TeV}$ can be discovered (excluded) with an integrated luminosity of $20 \mathrm{fb}^{-1}\left(5 \mathrm{fb}^{-1}\right)$.

- Things become even more interesting at $\sqrt{s}=14 \mathrm{TeV}$. The region of masses $M_{G} \sim 3-$ $4.5 \mathrm{TeV}$ can be discovered with an integrated luminosity of $\sim 5-100 \mathrm{fb}^{-1}$. Exclusion bounds in the case that no signal is discovered can go up to $M_{G} \sim 3.4,4,4.5$ and 5 $\mathrm{TeV}$ for integrated luminosities of $\sim 1,5,30$ and $100 \mathrm{fb}^{-1}$, respectively.

\section{2 $H j j$ channel: MFV scenario}
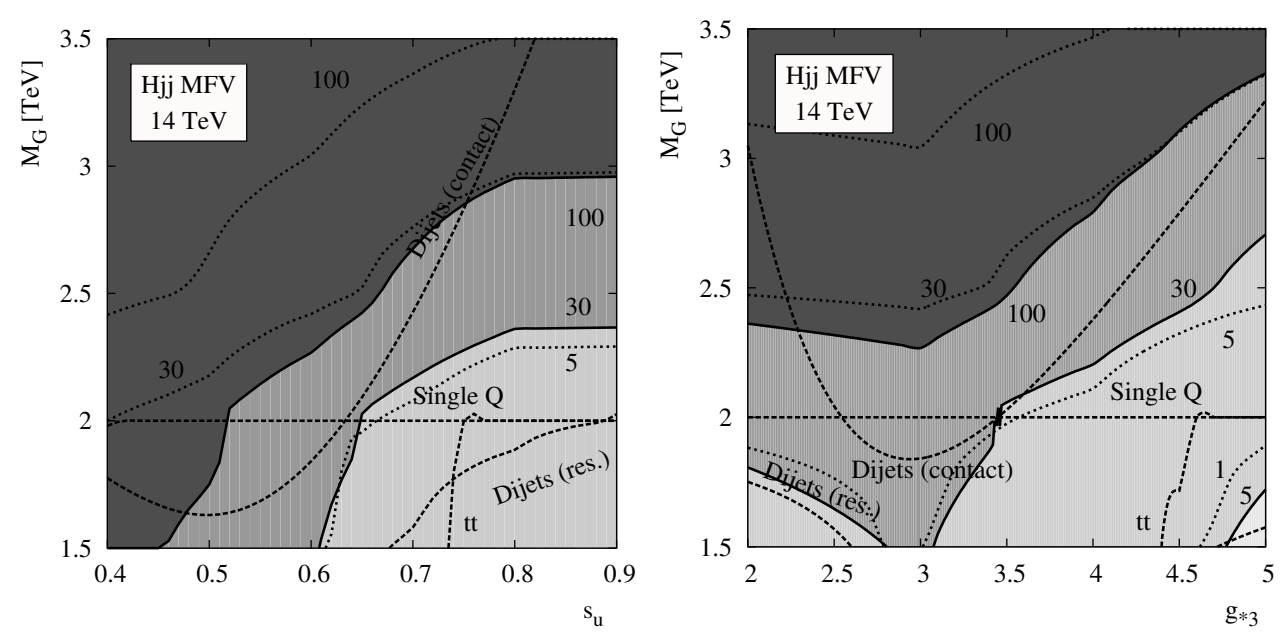

Figure 8. Contours of required luminosity for a $5 \sigma$ discovery (bands and solid lines) and $95 \%$ exclusion limits (dotted lines) as a function of $s_{u}$ and $M_{G}$ (left column) and $g_{* 3}$ and $M_{G}$ (right column) for $\sqrt{s}=14 \mathrm{TeV}$ in the $H j j$ channel (MFV scenario). Current bounds are shown with dashed lines (the area below the dashed lines is excluded).

The discovery and $95 \%$ bound contours for the $H j j$ channel as a function of $\left(s_{u}, M_{G}\right)$ and $\left(g_{* 3}, M_{G}\right)$ in the MFV scenario are given in Fig. 8. Because of the reduced statistics 
due to the leptonic decays of both $W$, this channel is not as promising as the $H t \bar{t}$ one. In fact, even with $\sqrt{s}=14 \mathrm{TeV}$, more than $30 \mathrm{fb}^{-1}$ of integrated luminosity are required for discovery in the allowed region of parameter space. With $100 \mathrm{fb}^{-1}$, masses up to $M_{G} \sim 3.3$ $\mathrm{TeV}$ can be discovered and up to $M_{G} \sim 3.5 \mathrm{TeV}$ excluded if no signal of new physics is observed. Thus, although this channel remains an important complementary test of the MFV scenario, it is likely that a much earlier signal of new physics would appear in other observables, like dijet or single vector-like production searches.

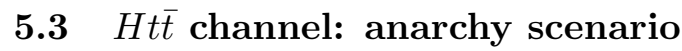
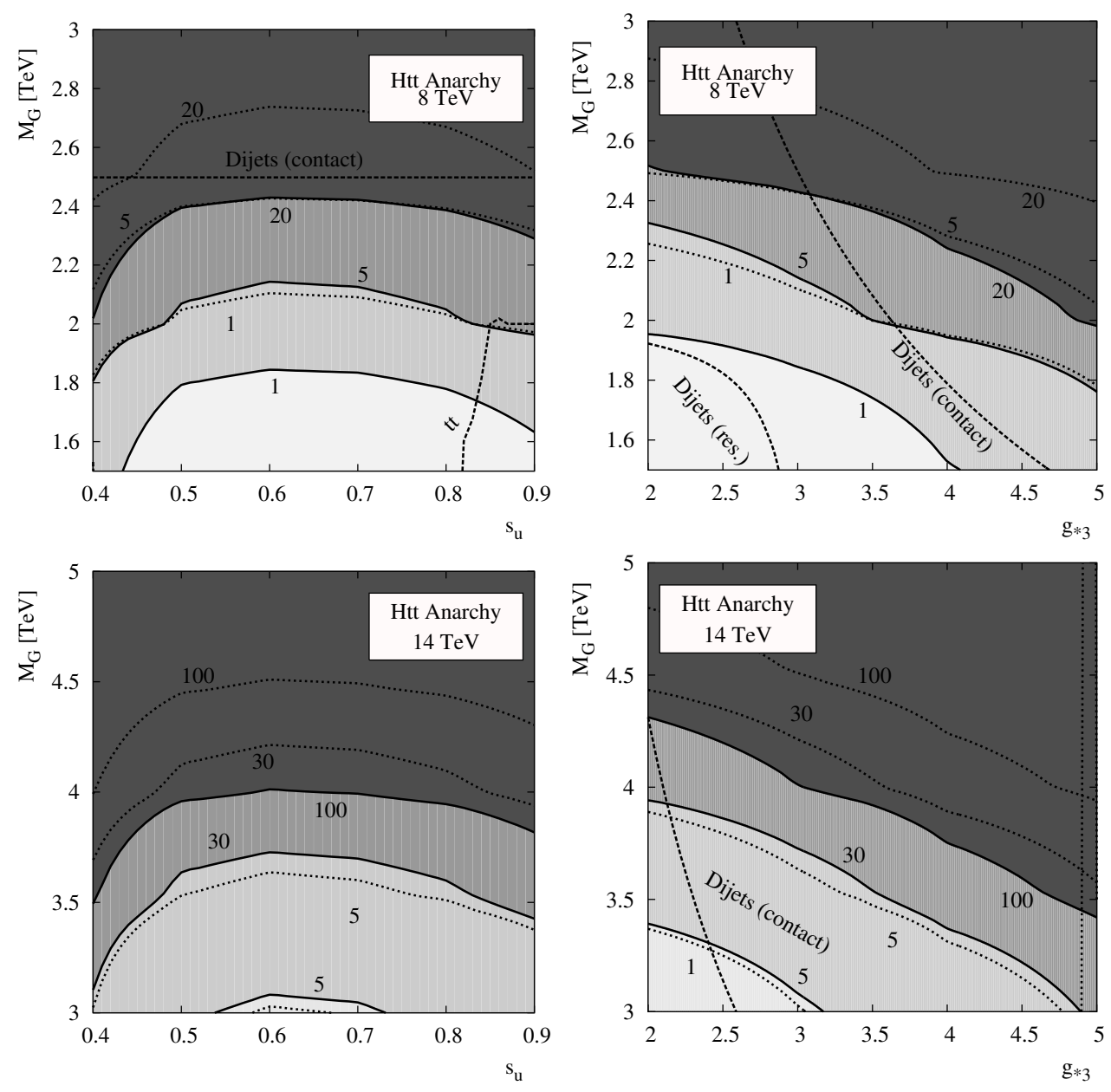

Figure 9. Contours of required luminosity for a $5 \sigma$ discovery (bands and solid lines) and $95 \%$ exclusion limits (dotted lines) as a function of $s_{u}$ and $M_{G}$ (left column) and $g_{* 3}$ and $M_{G}$ (right column) for $\sqrt{s}=8$ and $14 \mathrm{TeV}$ (first and second row, respectively) in the $H t \bar{t}$ channel (anarchy scenario). Current bounds are shown with dashed lines (the area below the dashed lines is excluded).

In the anarchic scenario, the light SM quarks are essentially elementary $\left(s_{u} \ll 1\right.$ for the first two generations). This has two main implications. First, the bounds from electroweak single production are irrelevant (the corresponding value of $\tilde{\kappa}$ is negligibly small). Second, 
the bounds from dijet contact interactions depend only on $g_{* 3}$. In particular, for the benchmark value $g_{* 3}=3$ they imply a constant bound $M_{G} \geq 2.5 \mathrm{TeV}$. This bound decreases as $g_{* 3}$ increases. For instance it becomes $M_{G} \geq 1.5 \mathrm{TeV}$ for $g_{* 3} \approx 4.6$. Our main results are shown in Fig. 9 and can be summarized in the following points:

- Using the 2011 run, masses up to $M_{G} \approx 1.9-1.6 \mathrm{TeV}$ can be discovered in the region allowed by current constraints for $g_{* 3} \gtrsim 4-5$. Exclusion bounds in the $M_{G} \sim 2.2-1.9$ $\mathrm{TeV}$ can be reached for $g_{* 3} \sim 3-5$. These results assume $s_{u} \sim 0.5-0.7$ (notice that in the anarchy case this refers to the $t_{R}$ degree of compositeness), outside this range, the reach decreases as shown in the left column of Fig. 9. The plot corresponding to this energy is not shown as it is quite similar to the one at $\sqrt{s}=8 \mathrm{TeV}$, only with the numbers reduced to match the results we have described.

- The expected 2012 run with $20 \mathrm{fb}^{-1}$ at $\sqrt{s}=8 \mathrm{TeV}$ can lead to a discovery in the region $M_{G} \sim 2.5-2 \mathrm{TeV}$ (and a similar exclusion with just $5 \mathrm{fb}^{-1}$ ) for $g_{* 3} \sim 3-5$. Exclusion bounds in the $M_{G} \sim 2.8-2.4 \mathrm{TeV}$ region can be set, for $g_{* 3} \sim 2.5-5$, with the same luminosity.

- Data with $\sqrt{s}=14 \mathrm{TeV}$ can probe a much larger region of parameter space. Values up to $M_{G} \sim 4.3 \mathrm{TeV}$ can be discovered with $100 \mathrm{fb}^{-1}$ and bounds up to $4.8 \mathrm{TeV}$ can be set with the same luminosity.

It is interesting to point out the differences between the anarchic and the MFV scenarios. In the former, discussed in this sub-section, the reach improves for intermediate values of $s_{u}$ (this is just due to the dependence of the GtT and Gt $\tilde{T}$ couplings) and for small values of $g_{* 3}$ (due to the larger coupling to valence quarks and therefore larger production cross section in that case). In the MFV case, on the other hand, the reach improves for larger values of $s_{u}$ (due to the larger coupling of $G$ to valence quarks and therefore to a larger production cross section) and for larger values of $g_{* 3}$ (due to a larger coupling of $G$ to $u_{R}$ and therefore to a larger production cross section). Thus, for instance in the benchmark models, the reach is slightly better in the anarchy model than in the MFV one (although the constraints from dijets are also stronger). The reach is even larger for smaller values of $g_{* 3}$ whereas it worsens quite a bit with respect to the MFV scenario for large values for $s_{u}$ or $g_{* 3}$.

\section{Conclusions}

The discovery of the Higgs boson and the measurement of its main properties have become the major goal of the ATLAS and CMS experiments for the 2012 run. Already the data collected during 2011 is starting to constrain many models of physics beyond the SM. We have shown that in models with a strong EWSB sector, the presence of new resonances of the composite sector can mediate new Higgs production mechanisms. In particular, single production of new vector-like quark resonances mediated by color octet vector resonances produce an $H t \bar{t}$ or $H j j$ final state that can be easily discovered at the LHC. Although these same final states are already present in the SM, their kinematical features and the 
couplings they depend on are completely different. For instance, the $H t \bar{t}$ channel is not directly related to the top Yukawa coupling, as happens in the SM contribution. Similarly, the two jets in the $H j j$ channel are quite hard and central, as opposed to vector-boson fusion Higgs production in the SM.

The experimental study of these new production mechanisms is important for two reasons. First, it uses the LHC community Higgs effort to explore ingredients of new models that go beyond the Higgs sector itself. Second, it shows that a re-analysis of channels already present in the SM but with fresh point of view can in some cases represent main discovery channels for physics beyond the SM.

We have found that masses for new color octet vector resonances up to $2.8 \mathrm{TeV}$ can be probed with the 2011 and 2012 data sets. This enters the region currently preferred by electroweak precision constraints. With the energy upgrade to $\sqrt{s}=14 \mathrm{TeV}$, up to $M_{G} \sim 5$ $\mathrm{TeV}$ can be probed with $100 \mathrm{fb}^{-1}$. This reach is comparable or even better than the one of more traditional searches [83, 84]. For masses above $2.5 \mathrm{TeV}$, boosted techniques have proven to be very efficient in extracting the signal. We have used a very simple analysis based on the invariant mass of fat jets but there is clearly room for improvement with the use of more sophisticated tools.

\section{Acknowledgments}

It is a pleasure to thank F. del Aguila, O. Domenech, C. Grojean, J. M. Lizana, P. Masjuan and M. Pérez-Victoria for useful discussions. This work has been supported by MICINN projects FPA2006-05294 and FPA2010-17915, through the FPU programme and by Junta de Andalucía projects FQM 101, FQM 03048 and FQM 6552.

\section{References}

[1] D. B. Kaplan and H. Georgi, SU(2) x U(1) Breaking by Vacuum Misalignment, Phys.Lett. B136 (1984) 183.

[2] D. B. Kaplan, H. Georgi, and S. Dimopoulos, Composite Higgs Scalars, Phys.Lett. B136 (1984) 187.

[3] S. Dimopoulos and J. Preskill, Massless composites with massive constituents, Nucl.Phys. B199 (1982) 206.

[4] K. Agashe and R. Contino, The Minimal composite Higgs model and electroweak precision tests, Nucl.Phys. B742 (2006) 59-85, [hep-ph/0510164].

[5] M. S. Carena, E. Ponton, J. Santiago, and C. E. Wagner, Light Kaluza Klein States in Randall-Sundrum Models with Custodial SU(2), Nucl.Phys. B759 (2006) 202-227, [hep-ph/0607106].

[6] M. S. Carena, E. Ponton, J. Santiago, and C. Wagner, Electroweak constraints on warped models with custodial symmetry, Phys.Rev. D76 (2007) 035006, [hep-ph/0701055].

[7] J. Espinosa, C. Grojean, and M. Muhlleitner, Composite Higgs Search at the LHC, JHEP 1005 (2010) 065, [arXiv:1003.3251]. 32 pages, 16 figures. 
[8] J. Espinosa, C. Grojean, and M. Muhlleitner, Composite Higgs under LHC Experimental Scrutiny, arXiv:1202.1286. 6 pages. Contribution to the proceedings of Hadron Collider Physics Symposium 2011, Paris Nov. 14-18.

[9] D. Carmi, A. Falkowski, E. Kuflik, and T. Volansky, Interpreting LHC Higgs Results from Natural New Physics Perspective, arXiv:1202.3144.

[10] A. Azatov, R. Contino, and J. Galloway, Model-Independent Bounds on a Light Higgs, arXiv: 1202.3415.

[11] J. Espinosa, C. Grojean, M. Muhlleitner, and M. Trott, Fingerprinting Higgs Suspects at the $L H C$, arXiv:1202.3697.

[12] R. Contino, "Hunting the composite higgs at the lhc." Talk given at the Workshop on Strongly Coupled Physics Beyond the Standard Model, ICPT, Trieste, January 26, 2012.

[13] R. Contino, C. Grojean, M. Moretti, F. Piccinini, and R. Rattazzi, Strong Double Higgs Production at the LHC, JHEP 1005 (2010) 089, [arXiv:1002.1011].

[14] N. Vignaroli, A New Strategy to Discover Heavy Colored Vectors at the Early LHC, arXiv: 1107.4558.

[15] R. Barcelo, A. Carmona, M. Masip, and J. Santiago, Stealth gluons at hadron colliders, Phys.Lett. B707 (2012) 88-91, [arXiv:1106.4054].

[16] R. Barcelo, A. Carmona, M. Chala, M. Masip, and J. Santiago, Single Vectorlike Quark Production at the LHC, Nucl.Phys. B857 (2012) 172-184, [arXiv:1110.5914].

[17] C. Bini, R. Contino, and N. Vignaroli, Heavy-light decay topologies as a new strategy to discover a heavy gluon, JHEP 1201 (2012) 157, [arXiv:1110.6058]. 36 pages, 13 figures. v2: a few typos corrected, comments added, version published in JHEP.

[18] B. A. Dobrescu, K. Kong, and R. Mahbubani, Prospects for top-prime quark discovery at the Tevatron, JHEP 0906 (2009) 001, [arXiv:0902.0792].

[19] G. Brooijmans, B. Gripaios, F. Moortgat, J. Santiago, P. Skands, et al., Les Houches 2011: Physics at TeV Colliders New Physics Working Group Report, arXiv:1203.1488.

[20] D. B. Kaplan, Flavor at SSC energies: A New mechanism for dynamically generated fermion masses, Nucl.Phys. B365 (1991) 259-278. Revised version.

[21] R. Contino, T. Kramer, M. Son, and R. Sundrum, Warped/composite phenomenology simplified, JHEP 0705 (2007) 074, [hep-ph/0612180].

[22] A. Falkowski and M. Perez-Victoria, Holographic Unhiggs, Phys.Rev. D79 (2009) 035005, [arXiv: 0810.4940].

[23] M. Redi and A. Weiler, Flavor and CP Invariant Composite Higgs Models, JHEP 1111 (2011) 108, [arXiv:1106.6357].

[24] F. del Aguila, G. L. Kane, and M. Quiros, A possible method to produce and detect Higgs bosons at hadron colliders, Phys.Rev.Lett. 63 (1989) 942.

[25] F. del Aguila, L. Ametller, G. L. Kane, and J. Vidal, Vector like fermion and standard higgs production at hadron colliders, Nucl.Phys. B334 (1990) 1.

[26] J. Aguilar-Saavedra, Light Higgs boson discovery from fermion mixing, JHEP 0612 (2006) 033, [hep-ph/0603200]. 
[27] J. Aguilar-Saavedra, Identifying top partners at LHC, JHEP 0911 (2009) 030, [arXiv: 0907.3155].

[28] G. D. Kribs, A. Martin, and T. S. Roy, Higgs boson discovery through top-partners decays using jet substructure, Phys.Rev. D84 (2011) 095024, [arXiv:1012.2866].

[29] A. Azatov, O. Bondu, A. Falkowski, M. Felcini, S. Gascon-Shotkin, et al., Higgs boson production via vector-like top-partner decays: diphoton or multilepton plus multijets channels at the LHC, arXiv:1204.0455.

[30] G. Azuelos, K. Benslama, D. Costanzo, G. Couture, J. Garcia, et al., Exploring little Higgs models with ATLAS at the LHC, Eur.Phys.J. C39S2 (2005) 13-24, [hep-ph/0402037].

[31] N. Vignaroli, Discovering the composite Higgs through the decay of a heavy fermion, arXiv: 1204.0468.

[32] K. Agashe, R. Contino, and A. Pomarol, The Minimal composite Higgs model, Nucl.Phys. B719 (2005) 165-187, [hep-ph/0412089].

[33] R. Contino, L. Da Rold, and A. Pomarol, Light custodians in natural composite Higgs models, Phys.Rev. D75 (2007) 055014, [hep-ph/0612048].

[34] A. Azatov and J. Galloway, Light Custodians and Higgs Physics in Composite Models, Phys.Rev. D85 (2012) 055013, [arXiv:1110.5646]. 31 pages, 8 figures.

[35] S. Casagrande, F. Goertz, U. Haisch, M. Neubert, and T. Pfoh, The Custodial Randall-Sundrum Model: From Precision Tests to Higgs Physics, JHEP 1009 (2010) 014, [arXiv: 1005.4315].

[36] A. Azatov, M. Toharia, and L. Zhu, Higgs Production from Gluon Fusion in Warped Extra Dimensions, Phys.Rev. D82 (2010) 056004, [arXiv:1006.5939].

[37] M. Carena, S. Casagrande, F. Goertz, U. Haisch, and M. Neubert, Higgs Production in a Warped Extra Dimension, arXiv: 1204.0008.

[38] CMS Collaboration, "A search using multivariate techniques for a standard model higgs boson decaying into two photons." CMS-PAS-HIG-12-001.

[39] CMS Collaboration, "Combination of SM, SM4, FP Higgs boson searches." CMS-PAS-HIG-12-008.

[40] G. Panico and A. Wulzer, The Discrete Composite Higgs Model, JHEP 1109 (2011) 135, [arXiv:1106.2719].

[41] S. De Curtis, M. Redi, and A. Tesi, The 4 D Composite Higgs, arXiv:1110.1613.

[42] C. Delaunay, O. Gedalia, S. J. Lee, G. Perez, and E. Ponton, Ultra Visible Warped Model from Flavor Triviality and Improved Naturalness, Phys.Rev. D83 (2011) 115003, [arXiv: 1007.0243].

[43] C. Delaunay, O. Gedalia, S. J. Lee, G. Perez, and E. Ponton, Extraordinary Phenomenology from Warped Flavor Triviality, Phys.Lett. B703 (2011) 486-490, [arXiv:1101.2902].

[44] M. Redi, Composite MFV and Beyond, arXiv:1203.4220.

[45] M. Carena, A. D. Medina, B. Panes, N. R. Shah, and C. E. Wagner, Collider phenomenology of gauge-Higgs unification scenarios in warped extra dimensions, Phys.Rev. D77 (2008) 076003, [arXiv:0712.0095]. 
[46] O. Matsedonskyi, G. Panico, and A. Wulzer, Light Top Partners for a Light Composite Higgs, arXiv:1204.6333.

[47] M. Redi and A. Tesi, Implications of a Light Higgs in Composite Models, arXiv:1205.0232.

[48] D. Marzocca, M. Serone, and J. Shu, General Composite Higgs Models, arXiv:1205.0770.

[49] J. Berger, J. Hubisz, and M. Perelstein, A Fermionic Top Partner: Naturalness and the LHC, arXiv:1205.0013.

[50] D. Dicus, B. Dutta, and S. Nandi, Top quark signature in extended color theories, Phys.Rev. D51 (1995) 6085-6091, [hep-ph/9412370].

[51] B. A. Dobrescu, K. Kong, and R. Mahbubani, Massive color-octet bosons and pairs of resonances at hadron colliders, Phys.Lett. B670 (2008) 119-123, [arXiv:0709.2378].

[52] B. Lillie, J. Shu, and T. M. Tait, Top Compositeness at the Tevatron and LHC, JHEP 0804 (2008) 087, [arXiv:0712.3057].

[53] K. Kumar, T. M. Tait, and R. Vega-Morales, Manifestations of Top Compositeness at Colliders, JHEP 0905 (2009) 022, [arXiv:0901.3808].

[54] G. Servant, Four-top events at the LHC, .

[55] M. Perelstein and A. Spray, Four boosted tops from a Regge gluon, JHEP 1109 (2011) 008, [arXiv:1106.2171].

[56] G. Cacciapaglia, R. Chierici, A. Deandrea, L. Panizzi, S. Perries, et al., Four tops on the real projective plane at LHC, JHEP 1110 (2011) 042, [arXiv:1107.4616]. 26 pages, 4 figures, minor modifications and few references added.

[57] J. Aguilar-Saavedra and J. Santiago, Four tops and the $t$ tbar forward-backward asymmetry, Phys.Rev. D85 (2012) 034021, [arXiv:1112.3778]. LaTeX 5 pages. Added results for 14 $\mathrm{TeV}$. Final version to appear in PRD.

[58] N. Zhou, D. Whiteson, and T. M. Tait, Limits on Four-Top Production from the ATLAS Same-sign Top-quark Search, arXiv:1203.5862.

[59] P. P. Giardino, K. Kannike, M. Raidal, and A. Strumia, Reconstructing Higgs boson properties from the LHC and Tevatron data, arXiv:1203.4254.

[60] A. Atre, M. Carena, T. Han, and J. Santiago, Heavy Quarks Above the Top at the Tevatron, Phys.Rev. D79 (2009) 054018, [arXiv: 0806.3966].

[61] A. Atre, G. Azuelos, M. Carena, T. Han, E. Ozcan, et al., Model-Independent Searches for New Quarks at the LHC, JHEP 1108 (2011) 080, [arXiv:1102.1987].

[62] F. del Aguila, A. Carmona, and J. Santiago, Tau Custodian searches at the LHC, Phys.Lett. B695 (2011) 449-453, [arXiv: 1007.4206].

[63] ATLAS Collaboration Collaboration, G. Aad et al., Search for heavy vector-like quarks coupling to light quarks in proton-proton collisions at sqrt(s) $=7 \mathrm{TeV}$ with the ATLAS detector, arXiv:1112.5755.

[64] G. H. Brooijmans, A. Delgado, B. A. Dobrescu, C. Grojean, M. Narain, et al., New Physics at the LHC: A Les Houches Report. Physics at Tev Colliders 2007-New Physics Working Group, arXiv:0802.3715.

[65] CMS Collaboration, "A Search for Resonances in Semileptonic Top Pair Production." CMS-PAS-TOP-11-009. 
[66] ATLAS Collaboration, "A Search for $t \bar{t}$ Resonances in the Dilepton Channel using 1.04 $\mathrm{fb}^{-1}$ pp Collisiions at $\sqrt{s}=7$ TeV." ATLAS-CONF-2011-123.

[67] ATLAS Collaboration, "A Search for $t \bar{t}$ Resonances in the Lepton Plus Jets Channel using $2.05 \mathrm{fb}^{-1}$ pp Collisions at $\sqrt{s}=7 \mathrm{TeV}$." ATLAS-CONF-2012-029.

[68] A. Pomarol and J. Serra, Top Quark Compositeness: Feasibility and Implications, Phys.Rev. D78 (2008) 074026, [arXiv:0806.3247].

[69] B. Lillie, J. Shu, and T. M. Tait, Kaluza-Klein Gluons as a Diagnostic of Warped Models, Phys.Rev. D76 (2007) 115016, [arXiv: 0706.3960].

[70] O. Domenech, A. Pomarol, and J. Serra, Probing the SM with Dijets at the LHC, arXiv: 1201.6510.

[71] ATLAS Collaboration Collaboration, G. Aad et al., Search for New Physics in Dijet Mass and Angular Distributions in pp Collisions at $\sqrt{s}=7 \mathrm{TeV}$ Measured with the ATLAS Detector, New J.Phys. 13 (2011) 053044, [arXiv: 1103.3864].

[72] CMS Collaboration Collaboration, S. Chatrchyan et al., Search for quark compositeness in dijet angular distributions from pp collisions at sqrt(s) $=7 \mathrm{TeV}$, arXiv:1202.5535.

[73] ATLAS Collaboration Collaboration, G. Aad et al., Search for New Physics in the Dijet Mass Distribution using $1 \mathrm{fb}^{-1}$ of pp Collision Data at $\sqrt{\mathrm{s}}=7 \mathrm{TeV}$ collected by the ATLAS Detector, Phys.Lett. B708 (2012) 37-54, [arXiv:1108.6311].

[74] J. Alwall, P. Demin, S. de Visscher, R. Frederix, M. Herquet, et al., MadGraph/MadEvent v4: The New Web Generation, JHEP 0709 (2007) 028, [arXiv:0706.2334].

[75] M. L. Mangano, M. Moretti, F. Piccinini, R. Pittau, and A. D. Polosa, ALPGEN, a generator for hard multiparton processes in hadronic collisions, JHEP 0307 (2003) 001, [hep-ph/0206293].

[76] T. Sjostrand, S. Mrenna, and P. Z. Skands, PYTHIA 6.4 Physics and Manual, JHEP 0605 (2006) 026, [hep-ph/0603175].

[77] S. Ovyn, X. Rouby, and V. Lemaitre, DELPHES, a framework for fast simulation of a generic collider experiment, arXiv:0903.2225. 36 pages, 13 figures, 7 tables. Corrected typos. Submitted to Comp. Phys. Comm.

[78] W. Skiba and D. Tucker-Smith, Using jet mass to discover vector quarks at the LHC, Phys.Rev. D75 (2007) 115010, [hep-ph/0701247].

[79] B. Holdom, t-prime at the LHC: The Physics of discovery, JHEP 0703 (2007) 063, [hep-ph/0702037].

[80] B. Holdom, The Heavy quark search at the LHC, JHEP 0708 (2007) 069, [arXiv:0705.1736].

[81] A. Abdesselam, E. B. Kuutmann, U. Bitenc, G. Brooijmans, J. Butterworth, et al., Boosted objects: A Probe of beyond the Standard Model physics, Eur.Phys.J. C71 (2011) 1661, [arXiv: 1012.5412].

[82] A. Altheimer, S. Arora, L. Asquith, G. Brooijmans, J. Butterworth, et al., Jet Substructure at the Tevatron and LHC: New results, new tools, new benchmarks, .

[83] K. Agashe, A. Belyaev, T. Krupovnickas, G. Perez, and J. Virzi, LHC Signals from Warped Extra Dimensions, Phys.Rev. D77 (2008) 015003, [hep-ph/0612015].

[84] B. Lillie, L. Randall, and L.-T. Wang, The Bulk RS KK-gluon at the LHC, JHEP 0709 (2007) 074, [hep-ph/0701166]. 